\title{
PD-I Blockade for Hepatocellular Carcinoma: Current Research and Future Prospects
}

\author{
Antonio D'Alessio (D) 1,2 \\ Lorenza Rimassa (iD) 2,3 \\ Alessio Cortellini ${ }^{1,4}$ \\ David James Pinato (D) ${ }^{1,5}$ \\ 'Department of Surgery \& Cancer, \\ Imperial College London, Hammersmith \\ Hospital, London, WI20HS, UK; \\ ${ }^{2}$ Department of Biomedical Sciences, \\ Humanitas University, Pieve Emanuele, \\ Milan, Italy; ${ }^{3}$ Medical Oncology and \\ Hematology Unit, Humanitas Cancer \\ Center, IRCCS Humanitas Research \\ Hospital, Rozzano, Milan, Italy; \\ ${ }^{4}$ Department of Biotechnology and \\ Applied Clinical Sciences, University of \\ L'Aquila, L'Aquila, Italy; ${ }^{5}$ Division of \\ Oncology, Department of Translational \\ Medicine, University of Piemonte \\ Orientale, Novara, Italy
}

\begin{abstract}
The treatment of hepatocellular carcinoma (HCC) has witnessed radical changes over the last few years, with the introduction of immune checkpoint inhibitors (ICI) in clinical practice, namely the combination of atezolizumab plus bevacizumab as the standard of care for first-line treatment of advanced HCC. The immunosuppressive microenvironment of the chronically inflamed liver makes HCC a fertile ground for the use of ICI. This review focuses on anti-programmed cell death-1 (PD-1) monoclonal antibodies (mAb), which have been extensively studied, as monotherapy, in combination with other ICI or with antiangiogenic agents. Currently, anti-PD-1 agents are approved by the United States Food and Drug Administration for second-line treatment in advanced HCC: nivolumab, alone or in combination with ipilimumab, and pembrolizumab. Lack of demonstration of survival benefit in first and second line led to the investigation of PD-1 agents in combination with multi-kinase inhibitors, with a number of first-line treatment regimens being actively investigated. Mounting evidence suggests a potential role of PD-1 blockade as adjuvant or neoadjuvant therapies. A key challenge remains the identification of biomarkers of response, since only a minority of patients appear to benefit from ICI.
\end{abstract}

Keywords: liver cancer, immunotherapy, advanced, immune checkpoint inhibition, PD-1, angiogenesis

\section{Introduction}

Liver cancer accounts for 830.180 deaths in the world every year and ranks 6th as most frequently diagnosed cancer and third as most frequent cause of cancer-related deaths. ${ }^{1}$ Hepatocellular carcinoma (HCC) represents the majority of cases of primary liver cancer. It arises most frequently in the context of an underlying liver disease, such as chronic viral hepatitis (hepatitis $\mathrm{C}$ virus [HCV] being the predominant etiological factor in Western countries and hepatitis B virus [HBV] in East Asia), alcoholic cirrhosis, non-alcoholic steatohepatitis (NASH), non-alcoholic fatty liver disease (NAFLD), and exposure to toxic agents such as aflatoxin. In particular, the incidence and the prevalence of NAFLD-related HCC are expected to increase, especially in the US, with NAFLD likely to become the most frequent etiology in the upcoming years. ${ }^{2}$ If diagnosed in the early phases, HCC can be potentially cured with surgical resection, liver transplantation, or loco-regional approaches. However, most of the patients are diagnosed with an advanced disease, and systemic agents represent the treatment of choice. ${ }^{3,4}$

The most frequently used staging system for HCC is the Barcelona Clinic Liver Cancer (BCLC) classification, and systemic treatment is indicated for patients
Correspondence: David James Pinato Department of Surgery \& Cancer Imperial College London, Hammersmith Hospital, Du Cane Road, London, WI2 OHS, UK

Tel +442083833720

Email david.pinato@imperial.ac.uk 
classified as BCLC C o BCLC B not deemed amenable to further loco-regional treatments. For more than a decade, multikinase inhibitors (MKIs) targeting the angiogenesis pathway have been the mainstay for advanced HCC treatment, with sorafenib being the only available first-line treatment, ${ }^{5,6}$ until another MKI, lenvatinib, was proven non-inferior to sorafenib in the Phase III REFLECT trial. $^{7}$ For patients progressing on or intolerant to sorafenib, several agents have been proven effective as secondline treatment. In particular, two MKIs, regorafenib and cabozantinib, achieved a significant survival advantage for sorafenib-pretreated patients in the phase III RESORCE and CELESTIAL trials, respectively. ${ }^{8,9}$ Of note, cabozantinib is the only agent showing a benefit as third-line treatment, since $27 \%$ of the enrolled patients in the CELESTIAL trial had received two previous lines of treatments. Ramucirumab, an anti-VEGF monoclonal antibody $(\mathrm{mAb})$, represents the only example of biomarker-driven therapy for HCC, since it showed to significantly prolong survival compared to placebo in sorafenib-pretreated patients with a baseline alfa-fetoprotein (AFP) $\geq 400 \mathrm{ng} /$ mL. ${ }^{10}$

However, the landscape of HCC treatment has dramatically changed in the last few years with the use of immune checkpoint inhibitors (ICI). The modulation of the immune system via the blockade of the programmed death-1 (PD-1)/PD-ligand 1 (PD-L1) and/or via the combined blockade of the cytotoxic T-lymphocytes antigen-4 (CTLA-4) has been approved for several cancer types, becoming the current standard of care in different lines of treatment. The implementation of ICI for HCC treatment has met several challenges, due to the fact that the presence of a chronic $\mathrm{HBV}$ or $\mathrm{HCV}$ infection has been classically considered as a contraindication to the use of immunotherapy, due to the fear of a viral reactivation. However, the presence of a largely immunotolerant environment in the liver makes the use of immunomodulatory agents for HCC particularly intriguing. ${ }^{11}$ The United States (US) Food and Drug Administration (FDA) approved the use of the combination of atezolizumab, an anti-PD-L1 mAb, plus bevacizumab, an anti-vascular endothelial growth factor (VEGF) $\mathrm{mAb}$, as first-line treatment of unresectable or metastatic HCC, after the positive results of the phase III IMbrave150 trial, where the combination outperformed sorafenib in terms of survival and response. ${ }^{12,13}$ For further lines of treatment, in sorafenibpretreated patients, the FDA has approved the use of nivolumab, an anti-PD-1, alone or in combination with ipilimumab, an anti-CTLA-4 mAb, and pembrolizumab, another anti-PD-1 agent (Table 1).

Table I Efficacy and Survival Outcomes of Anti-PD-I Agents in Pivotal Clinical Trials

\begin{tabular}{|c|c|c|c|}
\hline Outcome & $\begin{array}{c}\text { CheckMate } 040[28] \\
\text { Nivolumab }\end{array}$ & $\begin{array}{l}\text { KEYNOTE-240 [35] } \\
\text { Pembrolizumab }\end{array}$ & $\begin{array}{c}\text { CheckMate } 040 \text { [33] Nivolumab Plus } \\
\text { Ipilimumab }\end{array}$ \\
\hline Phase of the trial & Phase I/II & Phase III & Phase I/II \\
\hline Population & Sorafenib-pretreated patients & Sorafenib-pretreated patients & Sorafenib-pretreated patients \\
\hline $\begin{array}{l}\text { Confirmed ORR per } \\
\text { RECIST I.I, \% }\end{array}$ & 20 & 18.3 & 32 \\
\hline $95 \% \mathrm{Cl}$ & $(15-26)$ & $(14-23.4)$ & $(20-47)$ \\
\hline Complete response, $\mathrm{n}(\%)$ & $3(1)$ & $6(2.2)$ & $4(8)$ \\
\hline Partial response, n (\%) & $39(18)$ & $45(16.2)$ & $12(24)$ \\
\hline Stable disease, $\mathrm{n}(\%)$ & $96(45)$ & $122(43.9)$ & $9(18)$ \\
\hline \multicolumn{4}{|l|}{ Overall survival (months) } \\
\hline Median & NR & 13.9 & 22.8 \\
\hline $95 \% \mathrm{Cl}$ & - & $(11.6-16)$ & (9.4-NE) \\
\hline \multicolumn{4}{|l|}{$\begin{array}{l}\text { Progression-free survival } \\
\text { (months) }\end{array}$} \\
\hline Median & 4.0 & 3.0 & Not reported \\
\hline $95 \% \mathrm{Cl}$ & $(2.9-5.4)$ & $(2.8-4.1)$ & \\
\hline
\end{tabular}

Notes: ${ }^{a}$ Data from the arm $A$ of the trial; references are reported in square brackets.

Abbreviations: ORR, objective response rate; RECIST I.I, response evaluation criteria in solid tumors version I.I; $95 \% \mathrm{CI}, 95 \%$ confidence interval; $\mathrm{n}$, number of patients; NR, not reached; NE, not estimable. 
This review will focus on the use of PD- 1 inhibitors for the treatment of advanced HCC, with a special focus on the possible future application in earlier phases of the disease.

\section{The PD-I/PD-LI Pathway as a Therapeutic Actionable Driver of Anti-Cancer Immunity in HCC}

PD-1 and its ligand PD-L1 compose a widely studied checkpoint regulating the adaptive immune response. While PD-1 is a glycoprotein expressed on the cell membrane of tumor-infiltrating immune cells, PD-L1 is constitutively expressed on several lineages of immune cells and it is further upregulated upon their activation. It can also be expressed by tumor cells and by a number of somatic cells when exposed to proinflammatory cytokines, namely vascular endothelial cells, fibroblastic reticular cells, epithelia, pancreatic islet cells, astrocytes, and neurons. ${ }^{14}$ The PD-1/PD-L1 axis leads to a negative feedback on immune response, by blocking the T-cell receptor (TCR) and CD28 signaling. ${ }^{15,16}$ Immune escape is one of the hallmarks of cancer, ${ }^{17}$ and tumor cells often overexpress PD-L1 as a resistance mechanism, in order to hijack the PD-1/PD-L1 immunosuppressive pathway. For this reason, the development and the use of anti-PD-1 and anti-PD-L1 $\mathrm{mAb}$ has changed the treatment paradigm of many cancer types. HCC provides a particularly fertile ground to use ICI, since the underlying chronic disease induces an immune-tolerant microenvironment, where tumor cells can escape from immunological surveillance. An important function of the liver is to act as an "immunological gatekeeper" since it receives and metabolizes a number of immunologically active substances from the gut: in particular, patients with a liver disease are characterized by a "leaky gut", and the liver receives microbial components from the gut microbiota, including bacterial nucleic acid, lipopolysaccharides (LPS), and toxins, collectively referred to as microbial-associated molecular patterns (MAMPs) or pathogen-associated molecular patterns (PAMPs). ${ }^{18}$ HCC is known to escape from immune response thanks to aberrant expression of neoantigens and dysfunctional antigen presentation through the major histocompatibility complex. ${ }^{11}$ The impaired recruitment of CD4+ and cytotoxic CD8+ cells, combined with the expansion of tolerogenic dendritic cells and regulatory $\mathrm{T}$ cells (T-regs), contribute to the liver immune tolerance. Also, a number of cell lines play in role in the immunosuppressive microenvironment, namely myeloidderived suppressor cells (MDSCs), hepatic stellate cells, and tumor-associated macrophages (TAMs). ${ }^{11}$ Despite some initial fears that ICI could cause a viral flare in case of HCV or HBV-related HCC, the first pivotal trials unequivocally confirmed the safety of ICI in these patients, ${ }^{19}$ paving the way for the subsequent efficacy testing of immunotherapy in HCC. The first anti-PD-1 agent used in HCC was nivolumab, a fully human IgG4 mAb blocking PD-1 interaction with PD-L1 and its other ligand PD-L2, and then others followed, namely pembrolizumab, sintilimab, and tislelizumab.

In order to tackle the multiplicity of resistance mechanisms within the cancer-immunity interface, anti-PD-1 agents were tested in combination with other anti-tumor classes, such as anti-CTLA-4 mAb and antiangiogenic drugs. Double checkpoint blockade has showed to exert a synergistic anti-tumor effect across malignancies, in particular malignant melanoma ${ }^{20}$ and renal cell carcinoma. ${ }^{21}$ CTLA-4 is a receptor on the membrane of activated $\mathrm{T}$ cells and it is constitutively expressed on the membrane of CD4+ CD25+ regulatory $\mathrm{T}$ cells (T-regs). It binds to ligands of the B7 family (CD80/CD86) on antigen-presenting cells (APCs) and downplays the adaptive immune response, counteracting CD28 activating signal. ${ }^{22}$ The combined inhibition of PD- 1 and CTLA- 4 enhances CD8+ T cell-mediated tumoral killing, activating a broader gene response than with the single agents. ${ }^{23}$

Another avenue tested to promote synergistic efficacy is inhibition of the VEGF pathway, a master regulator of angiogenesis, immune tolerance and driver of poor prognosis in HCC. Anti-PD-1 agents have also been investigated in combination with anti-VEGF $\mathrm{mAb}$, such as bevacizumab, or with MKI with an antiangiogenic mechanism of action. The VEGF pathway is known to exert an immunosuppressive effect via a pleiotropic range of mechanisms, mainly involving the promotion of immunomodulating cell types (immature dendritic cells, myeloid-derived suppressor cells, tumor-associated macrophages, and T-regs) and the downregulation of CD8+ T cells activity. ${ }^{24}$ Moreover, VEGF promotes aberrant angiogenesis and local hypoxia, which is a known mechanism of tumor immune escape in $\mathrm{HCC}^{25}$ Preclinical data show that the use of anti-VEGF agents can revert these immunosuppressive effects, and the concomitant use of ICI can enhance the immune-mediated tumoral killing. ${ }^{26}$ Furthermore, the inhibition of the neoangiogenesis can restore the normal delivery of ICI in tumor 
tissues and can reduce the hypoxia-induced PD-L1 overexpression. $^{27}$

The presence of this strong preclinical rationale has paved the way for using ICIs as monotherapy or in combination in clinical practice for HCC treatment, translating into a significant benefit for cancer patients.

\section{Clinical Evidence for the Use of PD-I Inhibitors in Advanced HCC Anti-PD-I \pm Anti-CTLA-4 for Advanced Disease}

Nivolumab used as monotherapy was approved by the FDA for sorafenib-pretreated patients on the basis of the results of the phase I/II CheckMate-040 study. ${ }^{28}$ This was a multi-center, non-comparative, open-label trial testing the safety and efficacy of nivolumab in 48 patients for the dose-escalation and 214 for the dose-expansion phase. Patients were stratified according to viral and non-viral etiology and a Child-Pugh score up to B7 was allowed. The majority of patients had already been treated with sorafenib (182 out of $262,69.5 \%$ ). The recommended dose for the expansion phase was $3 \mathrm{mg} / \mathrm{kg}$ every 2 weeks, after exposing patients in the dose escalation phase to a dose from $0.1 \mathrm{mg} / \mathrm{kg}$ to $10 \mathrm{mg} / \mathrm{kg}$ without doselimiting toxicities. In the dose-expansion group, nivolumab achieved an objective response rate (ORR) of 20\% (95\% confidence interval $[\mathrm{CI}], 15-26)$ and a disease control rate (DCR) of 64\% (95\% CI, 58-71) per Response Evaluation Criteria in Solid Tumors (RECIST) 1.1 criteria, similar across the prespecified subgroups, and a 9-month overall survival rate of 74\% (95\% CI, 67-79) (Table 1). The experimental drug showed no unexpected safety signal, and in particular no viral flares in patients suffering from HCV and HBV hepatitis. Of note, nivolumab was proven a safe and effective option also in patients with a Child-Pugh B7 liver function, with a comparable rate of treatment-related adverse events (TRAEs) and treatment discontinuation due to an AE than in the general population. ${ }^{29}$ This pivotal trial paved the way for the CheckMate-459 trial, the multicenter phase III randomized trial testing the superiority of nivolumab in first line against the previous standard of care sorafenib. ${ }^{30}$ However, the study did not meet its primary endpoint: median OS did not significantly differ between the nivolumab arm (16.4 months [95\% CI, 13.9-18.4]) and the control arm (14.7 months [CI 95\%, 11.9-17.2]), with an HR of 0.85 (95\% CI, 0.72-1.02; $\mathrm{p}=0.0752$ ). Notably, both drugs achieved unprecedented results in terms of survival: nivolumab reached the highest median OS compared to the previous phase III first-line trials, and sorafenib obtained a much longer survival than in the SHARP $^{5}$ and in the Asia-Pacific ${ }^{6}$ trial, possibly reflecting a better patient selection and an improved AEs management. ${ }^{31}$ The authors highlighted a potential clinical benefit of nivolumab, with regard to ORR (15\% versus $7 \%$ for nivolumab and sorafenib, respectively), grade 3-4 (G3-4) TRAEs $(22 \%$ versus $49 \%$ ), and quality of life (QoL) in a further analysis. ${ }^{32}$ However, in view of the lack of survival advantage, nivolumab cannot be considered an alternative to sorafenib in first line.

The role of nivolumab in HCC was also explored in combination with ipilimumab. The double immune checkpoint blockade was investigated in the cohort 4 of the CheckMate-040 trial in patients who progressed on or were intolerant to sorafenib. ${ }^{33}$ The combination was tested at different dosing regimens: the best results were obtained in the arm A of this cohort, where 49 patients received nivolumab $1 \mathrm{mg} / \mathrm{kg}$ plus ipilimumab $3 \mathrm{mg} / \mathrm{kg}$ every 3 weeks for four doses, followed by single-agent nivolumab $240 \mathrm{mg}$ every 2 weeks. This regimen achieved an ORR of 32\% (95\% CI, 2047) per RECIST 1.1, including 4 complete responses (CR), and a median duration of response (DOR) not reached (NR) (Table 1). Also, the regimen in arm A outperformed the other arms in terms of survival, with a median OS of 22.8 months (95\% CI, 9.4-NR) versus 12.5 months (95\% CI, 7.6-16.4) and 12.7 months (95\% CI, 7.4-33.0) in arm B and C, respectively. In arm A, 53\% patients experienced a G3-4 TRAE, with the most frequent causes of treatment discontinuation being hepatitis $(6 \%)$, pneumonitis $(6 \%)$, colitis $(4 \%)$. Interestingly, researchers did not observe any recurrence of immunerelated $\mathrm{AE}$ (irAE) when patients suffering from an irAE were rechallenged with nivolumab or ipilimumab. Based on these results, the US FDA approved the use of the combination of nivolumab plus ipilimumab at the regimen tested in the arm A after sorafenib discontinuation. Also, this regimen is currently under investigation in the phase III CheckMate 9DW trial (NCT04039607), where it is tested in first line against sorafenib (Table 2). Another PD-1 inhibitor, pembrolizumab, received the FDA approval for sorafenib-pretreated patients after the positive results of the Phase II KEYNOTE-224 trial. ${ }^{34}$ The trial met its primary endpoint, with an ORR of $17 \%$ (95\% CI, 11-26), and prepared the ground for the subsequent randomized phase III KEYNOTE-240 trial. ${ }^{35}$ This trial randomized 413 patients who had failed prior sorafenib in a 2:1 ratio to pembrolizumab or to placebo. Due to the statistical design, which established a prespecified boundary of $\mathrm{p}=0.0174$ for OS, the primary endpoint, median OS, was not met: pembrolizumab achieved a median OS of 13.9 months 
Table 2 Ongoing Phase III Trials with Anti-PD-I Agents for First-Line Advanced Disease

\begin{tabular}{|c|c|c|c|c|c|c|}
\hline \multirow{2}{*}{$\begin{array}{l}\text { Target } \\
\text { Population }\end{array}$} & \multicolumn{6}{|c|}{$\begin{array}{l}\text { Confirmed Pathologic or Radiological }{ }^{\mathrm{a}} \text { Diagnosis of HCC; BCLC B Unsuitable for Locoregional Therapy or } \\
\text { BCLC C; No Prior Systemic Therapy for HCC; Child-Pugh Class A; ECOG PS 0-I. }\end{array}$} \\
\hline & Phase & Study Regimens & $\begin{array}{l}\text { Estimated } \\
\text { Sample } \\
\text { Size }\end{array}$ & Status & $\begin{array}{l}\text { Primary } \\
\text { Endpoints }\end{array}$ & Secondary Endpoints \\
\hline \multirow{2}{*}{$\begin{array}{l}\text { RATIONALE- } \\
30 \mathrm{I}^{\mathrm{b}}[36] \\
\text { NCT034I } 2773\end{array}$} & \multirow[t]{2}{*}{ III } & Tislelizumab $200 \mathrm{mg}$ iv q3w & \multirow[t]{2}{*}{674} & \multirow{2}{*}{$\begin{array}{l}\text { Active, } \\
\text { not } \\
\text { recruiting }\end{array}$} & \multirow[t]{2}{*}{ OS } & \multirow{2}{*}{$\begin{array}{l}\text { ORR, PFS, DOR, TTP, HRQOL, DCR, } \\
\text { CBR }\end{array}$} \\
\hline & & Sorafenib $(800 \mathrm{mg} /$ day $)$ & & & & \\
\hline \multirow[t]{2}{*}{$\begin{array}{l}\text { LEAP-002 [40] } \\
\text { NCT037I3593 }\end{array}$} & \multirow[t]{2}{*}{ III } & $\begin{array}{l}\text { Pembrolizumab } 200 \mathrm{mg} \text { iv q } 3 \mathrm{w} \text { plus } \\
\text { Lenvatinib ( } 12 \mathrm{mg} / \text { day or } 8 \mathrm{mg} / \text { day } \\
\text { according to body weight } \geq \text { or }< \\
60 \mathrm{~kg} \text { ) }\end{array}$ & \multirow[t]{2}{*}{750} & \multirow[t]{2}{*}{$\begin{array}{l}\text { Active, } \\
\text { not } \\
\text { recruiting }\end{array}$} & \multirow[t]{2}{*}{ PFS, OS } & \multirow[t]{2}{*}{$\begin{array}{l}\text { ORR, DOR, DCR and TTP both per } \\
\text { RECIST I.I and mRECIST, PFS per } \\
\text { mRECIST, lenvatinib pharmacokinetics, } \\
\text { safety }\end{array}$} \\
\hline & & Lenvatinib plus a matching-placebo & & & & \\
\hline \multirow[t]{2}{*}{$\begin{array}{l}\text { CheckMate } \\
\text { 9DW } \\
\text { NCT04039607 }\end{array}$} & \multirow[t]{2}{*}{ III } & $\begin{array}{l}\text { Nivolumab I mg/kg plus Ipilimumab } \\
3 \mathrm{mg} / \mathrm{kg} \text { × } 4 \mathrm{q} 3 \mathrm{w} \text { followed by } \\
\text { Nivolumab } 480 \mathrm{mg} \mathrm{q} 4 \mathrm{w}\end{array}$ & \multirow[t]{2}{*}{1084} & \multirow[t]{2}{*}{ Recruiting } & \multirow[t]{2}{*}{ OS } & \multirow[t]{2}{*}{ ORR, DOR, TTSD } \\
\hline & & $\begin{array}{l}\text { Sorafenib }(800 \mathrm{mg} / \text { day) or Lenvatinib } \\
(12 \mathrm{mg} / \text { day or } 8 \mathrm{mg} / \text { day according to } \\
\text { body weight } \geq \text { or }<60 \mathrm{~kg} \text { ) }\end{array}$ & & & & \\
\hline
\end{tabular}

Notes: ${ }^{a}$ A radiological diagnosis according to the American Association for the Study of Liver Diseases (AASLD) criteria is admitted in LEAP-002 trials for cirrhotic patients; ${ }^{\mathrm{b}}$ non inferiority trial; references are reported in square brackets.

Abbreviations: HCC, hepatocellular carcinoma; BCLC, Barcelona Clinic Liver Cancer; ECOG PS, Eastern Cooperative Oncology Group Performance Status; iv, intravenously; q3w, every 3 weeks; OS, overall survival; ORR, objective response rate; PFS, progression-free survival; DOR, duration of response; TTP, time to progression; HRQoL, health-related quality of life; DCR, disease control rate; CBR, clinical benefit rate; RECIST I.I, response evaluation criteria in solid tumors version I.I; mRECIST, modified response evaluation criteria in solid tumors; q4w, every 4 weeks; TTSD, time to symptom deterioration.

(95\% CI, 11.6-16.0) versus 10.6 months (95\% CI, 8.3-13.5) with placebo (HR 0.78; 95\% CI, 0.611-0.998; $\mathrm{p}=0.0238$ ), while median progression-free survival (PFS) was 3.0 months (95\% CI, 2.8-4.1) versus 2.8 months (95\% CI, 1.6-3.0) with pembrolizumab and placebo, respectively (HR, $0.72 ; 95 \% \mathrm{CI}$, $0.570-0.904 ; p=0.0022$, where the prespecified boundary was $\mathrm{p}=0.002$ ) (Table 1). Despite the consistency of these findings with the previous phase II study, this trial was formally negative. After the disappointing findings of anti-PD-1 mAbs as monotherapy, it will be of high interest to analyze the data from the phase III non-inferiority trial (RATIONALE 301: NCT03412773) ${ }^{36}$ currently testing tislelizumab (BGB-A317) , another anti-PD-1 mAb, against sorafenib in first line, after the promising results obtained in a previous phase $\mathrm{Ia} / \mathrm{b}$ trial $^{37}$ (Table 2).

\section{Anti-PD-I Plus Antiangiogenic Agents}

Following the success of the combination of atezolizumab and bevacizumab in the IMBrave150 study, ${ }^{12}$ another combination was proven effective in treatment-naïve HCC patients. The ORIENT-32 was a phase II/III trial testing the superiority of the combination of sintilimab, an anti-PD-1 $\mathrm{mAb}$, and a bevacizumab biosimilar against sorafenib as first-line treatment in a Chinese, HBV-predominant population. ${ }^{38}$ The trial reached its primary endpoints, both median OS (not estimable [NE] versus 10.4 months; HR 0.57; 95\% CI, 0.43-0.75; $\mathrm{p}<0.0001)$ and median PFS (4.6 versus 2.8 months; HR 0.56 ; 95\% CI 0.45-0.7; p <0.0001), thus making the combination a possible new standard of care for Chinese patients. Earlier phase studies tested the combination of anti-PD-1 mAb and MKI with a known antiangiogenic spectrum. Pembrolizumab plus lenvatinib was administered in a phase $\mathrm{Ib}$ study to 100 untreated $\mathrm{HCC}$ patients, with an ORR of $46.0 \%(95 \% \mathrm{CI}, 36.0-56.3)$ and a DCR of $88.0 \%$ (95\% CI, 80.0-93.6) per modified RECIST (mRECIST) criteria by independent review. ${ }^{39}$ Based on these results, a phase III trial is currently testing the combination in a large population against sorafenib ${ }^{40}$ (Table 2). Lenvatinib has been successfully combined with nivolumab in another phase Ib study conducted in 30 Japanese patients, where the combination obtained a promising ORR of $76.7 \%$ per mRECIST criteria. ${ }^{41}$ Moreover, a third phase $\mathrm{Ib}$ trial 
tested the combination of pembrolizumab plus regorafenib on 32 treatment-naïve patients, with an interesting ORR of $28 \%$ and a manageable safety profile. ${ }^{42}$ Another anti-PD-1 agent, camrelizumab, was combined with apatinib (antiVEGFR-2 MKI) in the phase II RESCUE trial, where the combination was tested both in first and second line, achieving an ORR of $34 \%$ for untreated patients and $22.5 \%$ as second line. ${ }^{43}$

\section{Clinical Evidence for the Use of PD-I Inhibitors in Earlier Phases} Anti-PD-I as Adjuvant Therapy for HCC Currently, no treatment has proved to be effective in the adjuvant setting for $\mathrm{HCC}$, after surgical resection, liver transplantation, or loco-regional treatment. These approaches can be performed for patients diagnosed with early-stage disease with a curative intent; however, tumor recurrence affects long-term survival, with relapse rates of up to $70 \%$ and 5 -year OS $<50 \%{ }^{44}$ Early relapse involves the recurrence of the primary tumor, while late relapse involves the appearance of a new primary tumor in the context of a chronically damaged liver. The only phase III trial testing a possible adjuvant treatment for HCC was the STORM trial, failing to demonstrate an advantage in relapse-free survival with sorafenib. ${ }^{45}$ Anti-PD-1 mAb have already proved effective in the adjuvant setting in other cancer types, such as melanoma ${ }^{46}$ and gastroesophageal cancer, ${ }^{47}$ showing a significant survival advantage. For HCC, several immunotherapy strategies have already been tried, such as cancer vaccines ${ }^{48}$ and lymphocyte infusions, ${ }^{49}$ without conclusive evidence. Currently, large phase III clinical trials are investigating the role of anti-PD $-1 \mathrm{mAb}$ in the adjuvant setting after curative resection or ablation, in particular nivolumab (CheckMate 9DX: NCT03383458) and pembrolizumab (KEYNOTE-937: NCT03867084). However, due to the high risk of acute graft rejection and due to the need of a chronic immunosuppressive therapy, it is not possible to design a trial with an adjuvant ICI after a liver transplantation. ${ }^{50}$

\section{Anti-PD-I as Neoadjuvant Therapy}

Most of HCC patients are diagnosed at a late stage of the disease, when it is not possible to offer a potentially curative treatment, such as surgical resection, liver transplantation or local ablation. For this reason, the use of a systemic therapy as a bridge to downstage the disease and to make it susceptible of a loco-regional approach is an appealing strategy. If this strategy was difficulty viable in the past, given the low ORR obtained by MKI, the more robust responses seen in the ICI clinical trials bring the spotlight on the neoadjuvant approach for HCC. Furthermore, the use of ICI in the neoadjuvant setting offers a unique possibility of observing the in vivo response to the checkpoint blockade, thus providing precious tissue samples for biomarker studies. Currently, no clinical data are available of the efficacy of anti-PD-1 in this setting, but several phase I/II clinical trials are currently testing nivolumab (NCT03630640), cemiplimab (NCT03916627), and toripalimab (NCT03867370). Moreover, a phase II study (NCT03630640) and the phase Ib PRIME-HCC trial ${ }^{51}$ (NCT03682276) are investigating the use of the combination of nivolumab and ipilimumab prior to surgical resection, and the latter will provide a thorough biomarker study on biological samples.

\section{Anti-PD-I in Combination with Loco-Regional Treatments}

Loco-regional treatments are the treatment of choice for early and intermediate stage HCC. ${ }^{3}$ Besides their classic ablative action, a growing evidence points at the possible immunomodulatory role of these techniques, thus suggesting a potential synergism with immune checkpoint blockade. In particular, radiofrequency ablation (RFA) and microwave ablation (MWA), used for early-stage HCC, are known to influence the tumor microenvironment via the release of tumor neoantigens, thus promoting local infiltration of CD8+ T cells and the systemic CD8+ T cellmediated response. ${ }^{52}$ Also, the well-known abscopal effect induced by radiotherapy (RT) has not been characterized yet in HCC, but, considering the growing use of stereotactic body RT in early-stage HCC, the combination with ICI could be of high interest. ${ }^{53}$ Trans-arterial chemoembolization (TACE) is the treatment of choice for BCLC $\mathrm{B}$ stage HCC. The ischemic damage caused by the arterial occlusion induces the production of the hypoxia inducible factor (HIF)-1 $\alpha$, a known modulator of PD-L1 expression. Furthermore, TACE can act on tumor microenvironment by decreasing the percentage of T-regs and increasing the CD4+/CD8+ ratio, thus suggesting a possible positive interaction with ICI anti-tumor activity. ${ }^{54}$ This rationale guided the design of trials testing the combination of TACE and anti-PD-1 mAb, for instance the phase I/II PETAL study is investigating the safety and preliminary efficacy of pembrolizumab after TACE in intermediate 
stage HCC (NCT03397654), while another study is testing the combination of nivolumab plus drug eluting beadTACE (deb-TACE) (NCT03143270).

\section{Biomarkers of Response}

Despite the major advances in the use of immunotherapy for HCC, only a minority of patients respond to ICIs. The causes beneath the lack of response are not fully understood, and the search for biomarkers of response has become an area of high unmet need. ${ }^{55}$ Biomarkers associated with ICI efficacy in other cancer types appear to have little utility in HCC. For instance, microsatellite instability (MSI) is a widely recognized biomarker of response to ICI, and FDA granted pembrolizumab a siteagnostic accelerated approval for any MSI-high (MSI-H) or mismatch-repair deficient (dMMR) unresectable or metastatic cancer type, based on the response rates in the KEYNOTE-016 study. ${ }^{56}$ However, the percentage of MSI$\mathrm{H}$ among HCC patients is low $(<3 \%)^{57}$ and, despite some anecdotal evidence, ${ }^{58,59}$ the testing for MMR status as biomarker of response for ICI in HCC would be of low impact. Tumor mutational burden (TMB) over ten mutations per megabase (defined as TMB-high [TMB-H]) was found to be associated with a response to ICI in the KEYNOTE-158 study, ${ }^{60}$ leading the FDA to grant pembrolizumab its second site-agnostic approval for the treatment of unresectable or metastatic TMB-H cancer regardless of its primary site. However, the study did not include any HCC patient, and HCC itself normally arises in an immunosuppressive microenvironment, with cases of TMB-H HCC being infrequent. ${ }^{61}$ PD-L1 expression, measured with different immunohistochemistry assays either on tumor cells as tumor proportional score (TPS) either on tumor and surrounding immune cells as combined positive score (CPS), is a predictive biomarker of response in several cancer types. ${ }^{62}$ However, the efficacy of ICIs in $\mathrm{HCC}$ does not seem to be related to PD-L1 expression. For instance, in the CheckMate-040 trial, the PD-L1 expression on tumor cells was assessed as secondary endpoint, but response to nivolumab was comparable across subgroups (PD-L1 < or $\geq 1 \%$ ). ${ }^{28}$ However, in a preplanned exploratory analysis of the KEYNOTE-224 trial, the response to pembrolizumab seemed to be linked to PDL1 expression quantified with CPS, but not with TPS, ${ }^{34}$ suggesting that further, prospective studies are needed to clarify the issue. Currently, the FDA approvals for ICIs HCC do not take into account the PD-L1 expression: the measure of PD-L1 is not performed in clinical practice and the lack of PD-L1 expression is not an exclusion criterion for the administration of ICIs. On the other hand, a more integrated evaluation of tumor microenvironment and immune phenotypes could be crucial to better predict ICI response. ${ }^{63,64}$ A post-hoc biomarker analysis performed on the tissues of patients treated with nivolumab within the dose-escalation and dose-expansion phases of the CheckMate-040 identified a possible role of a 4-gene signature as predictor of response to ICI. ${ }^{65}$ This analysis further highlighted the limitation of considering PD-L1 expression as a sole biomarker, since responses to nivolumab were registered regardless of PD-L1 expression, even if ORR were numerically higher in PD-L1 $\geq 1 \%$ patients.

Recent data show that response to ICI could depend on HCC aetiology. In particular, patients who develop HCC on the background of NASH etiology have been found to harbour an enriched population of exhausted CD8+PD1+ T cells. ${ }^{66}$ These aberrant cells are linked to an impaired immune surveillance, leading to a lack of response to ICI. This preclinical observation has been further corroborated by a meta-analysis of three randomized phase III clinical trials investigating ICI in advanced HCC, showing that survival of non-viral HCC patients was not improved by immunotherapy. ${ }^{66}$ Prospective evaluation of these mechanisms is warranted; however, immunotherapy still remains a valid treatment regardless of the underlying aetiology. $^{67}$

Additional data will be provided by the biomarker analyses planned within the ongoing phase III immunotherapy trials, both on tumor samples and other biological samples to overcome the problem of biopsy availability, trying to disentangle the complicated issue of ICI response in HCC.

\section{Open Challenges}

In 2017, the first results of the CheckMate-040 trial showed that anti-PD-1 inhibitors could radically change the natural history of $\mathrm{HCC}$, challenging the dominance of MKI in the treatment algorithm. ${ }^{28}$ These pivotal findings paved the way, a couple of years later, to the unquestionable success of the anti-PD-L1 mAb atezolizumab in combination with bevacizumab in the IMbrave150 study. ${ }^{12}$ However, the disappointing data coming from the use of nivolumab ${ }^{30}$ and pembrolizumab ${ }^{35}$ as monotherapy raise an intricate question: is there still a place for anti-PD-1 agents in HCC? Undoubtedly, despite the trial not reaching its primary endpoint, Checkmate-459 highlighted the good safety profile of nivolumab, and its better 
tolerability compared to sorafenib. ${ }^{32}$ We can speculate that a possible role of anti-PD-1 agents as monotherapy could be based on the favorable impact on QoL. Based on the trial results, nivolumab cannot be considered as standard of care for first line treatment. Perhaps a non-inferiority trial focused on adverse events and QoL could carve out a niche for PD-1-based immunotherapies in the complex treatment landscape of HCC. However, the number of patients required for a large non-inferiority trial and the issues related to this type of trial could discourage from planning one. ${ }^{68}$ Currently, a phase III non-inferiority trial (RATIONALE 301: NCT03412773) is testing an anti-PD $-1 \mathrm{mAb}$, tislelizumab, against sorafenib. ${ }^{36}$ Whilst atezolizumab plus bevacizumab have become standard of care, the results of the trial may still change first-line treatment choices. PD-1 monotherapy holds the benefit of being safe and be characterized by a lower proportion of long-term adverse events such as diarrhea, skin toxicity and fatigue typical of MKIs, which may limit patient's QoL. Also, nivolumab is the only agent proved safe and effective in Child B patients in clinical trials ${ }^{29}$ and post-registration studies, ${ }^{69}$ so further, prospective data on larger populations could provide important clinical data on the positioning of anti-PD-1 therapy agent in this more fragile segment of HCC patients, for which no approved therapy exists. The continued use of pembrolizumab as a second-line therapy is also debatable in view of atezolizumab and bevacizumab having become standard of care in first line and lack of survival benefit proven in the KEYNOTE-240 trial. It appears unlikely that pembrolizumab will be approved outside FDA territory in view of lack of OS benefit. ${ }^{70}$ This is unfortunate as a number of patients who may not be eligible to atezolizumab and bevacizumab could still benefit from PD-1 inhibition as a second-line therapy. An area of active investigation is the a priori identification of the subset of patients benefitting from pembrolizumab after sorafenib failure. However, clinical trial datasets have been unhelpful in answering this question. Some hints could possibly come from the identification of molecular signatures predicting response to immunotherapybased approach. For instance, nearly $25 \%$ of HCC fall into the "immune class" category, showing the markers of a potential better response to ICIs. ${ }^{64}$ On the other side, negative biomarkers found in preclinical models could also be highly useful to exclude patients from unnecessary and potentially toxic treatments. ${ }^{71}$ It is therefore likely that anti-PD-1/PD-L1 blockade will keep its position in the HCC treatment algorithm in combination with other agents, since the preliminary results of the phase I/II studies ${ }^{39,41,42}$ are highly promising and the phase III trials on their use in first line are currently ongoing. Furthermore, the possible applications of anti-PD-1 agents in earlier stages of the disease, as in the neo-/adjuvant setting or in combination with loco-regional treatments, is intriguing and could expand the domain of immunotherapy use in HCC. ${ }^{72,73}$ In particular, the immunomodulatory role of ablation, SBRT, and TACE makes the results of the ongoing studies focusing on the combination with ICI eagerly awaited. In case of a relapse after early use of antiPD-1 agents, it will be challenging to address the role of atezolizumab plus bevacizumab or other combinations in advanced/relapsed disease, since the evidence supporting the use of ICI in immunotherapy-pretreated patients is growing for other solid tumors ${ }^{74,75}$ but still lacking for HCC. The growing number of phase III clinical trials due to report in the next few years will be crucial in further reshaping the treatment landscape of HCC. While cancer immunotherapy continues to expand in clinical research and routine practice, the revolutionizing role of anti-PD-1/ PD-L1 agents as a backbone to novel immunotherapeutic combination approaches is undoubted. As such, their clinical use, with different indications and in combination, is likely to expand.

\section{Disclosure}

$\mathrm{AD}$ is supported by grant funding from the European Association for the Study of the Liver (Andrew Burroughs Fellowship). DJP received lecture fees from ViiV Healthcare and Bayer Healthcare and travel expenses from BMS and Bayer Healthcare; consulting fees for H3B, Mina Therapeutics, EISAI, Roche, and Astra Zeneca; received research funding (to institution) from MSD and BMS. DJP is also supported by grant funding from the Wellcome Trust Strategic Fund (PS3416) and acknowledges grant support from the Cancer Treatment and Research Trust (CTRT) and infrastructural support by the Cancer Research UK Imperial Centre and the NIHR Imperial Biomedical Research Centre. AC received consulting fees from MSD, BMS, AstraZeneca, Roche; speakers' fee from AstraZeneca, MSD, Novartis and Astellas. LR received consulting fees from Amgen, ArQule, AstraZeneca, Basilea, Bayer, BMS, Celgene, Eisai, Exelixis, Genenta, Hengrui, Incyte, Ipsen, IQVIA, Lilly, MSD, Nerviano Medical Sciences, Roche, Sanofi, Zymeworks; lecture fees from AbbVie, Amgen, Bayer, Eisai, Gilead, Incyte, Ipsen, Lilly, Merck Serono, Roche, Sanofi; travel expenses from Ipsen; and institutional 
research funding from Agios, ARMO BioSciences, AstraZeneca, BeiGene, Eisai, Exelixis, Fibrogen, Incyte, Ipsen, Lilly, MSD, Nerviano Medical Sciences, Roche, Zymeworks. The authors have no other relevant affiliations or financial involvement with any organization or entity with a financial interest in or financial conflict with the subject matter or materials discussed in the manuscript apart from those disclosed. No writing assistance was utilized in the production of this manuscript. The authors are fully responsible for all content and editorial decisions.

\section{References}

1. International Agency for Research on Cancer. The global cancer observatory. 2020; https://gco.iarc.fr/today/data/factsheets/cancers/ 11-Liver-fact-sheet.pdf. Accessed May 18, 2021.

2. Estes C, Razavi H, Loomba R, Younossi Z, Sanyal AJ. Modeling the epidemic of nonalcoholic fatty liver disease demonstrates an exponential increase in burden of disease. Hepatology. 2018;67:123-133. doi:10.1002/hep. 29466

3. Forner A, Reig M, Bruix J. Hepatocellular carcinoma. Lancet. 2018;391(10127):1301-1314. doi:10.1016/S0140-6736(18)30010-2

4. Park JW, Chen M, Colombo M, et al. Global patterns of hepatocellular carcinoma management from diagnosis to death: the BRIDGE study. Liver Int. 2015;35(9):2155-2166. doi:10.1111/liv.12818

5. Llovet JM, Ricci S, Mazzaferro V, et al. Sorafenib in advanced hepatocellular carcinoma. $N$ Engl J Med. 2008;359(4):378-390. doi:10.1056/NEJMoa0708857

6. Cheng AL, Kang YK, Chen Z, et al. Efficacy and safety of sorafenib in patients in the Asia-Pacific region with advanced hepatocellular carcinoma: a phase III randomised, double-blind, placebo-controlled trial. Lancet Oncol. 2009;10(1):25-34. doi:10.1016/S1470-2045(08)70285-7

7. Kudo M, Finn RS, Qin S, et al. Lenvatinib versus sorafenib in first-line treatment of patients with unresectable hepatocellular carcinoma: a randomised Phase 3 non-inferiority trial. Lancet. 2018;391 (10126):1163-1173. doi:10.1016/S0140-6736(18)30207-1

8. Bruix J, Qin S, Merle P, et al. Regorafenib for patients with hepatocellular carcinoma who progressed on sorafenib treatment (RESORCE): a randomised, double-blind, placebo-controlled, phase 3 trial. Lancet. 2017;389(10064):56-66. doi:10.1016/S0140-6736(16)32453-9

9. Abou-Alfa GK, Meyer T, Cheng AL, et al. Cabozantinib in patients with advanced and progressing hepatocellular carcinoma. N Engl J Med. 2018;379(1):54-63. doi:10.1056/NEJMoa1717002

10. Zhu AX, Kang YK, Yen CJ, et al. Ramucirumab after sorafenib in patients with advanced hepatocellular carcinoma and increased alpha-fetoprotein concentrations (REACH-2): a randomised, double-blind, placebo-controlled, phase 3 trial. Lancet Oncol. 2019;20(2):282-296. doi:10.1016/S1470-2045(18)30937-9

11. Flynn MJ, Sayed AA, Sharma R, Siddique A, Pinato DJ. Challenges and opportunities in the clinical development of immune checkpoint inhibitors for hepatocellular carcinoma. Hepatology. 2019;69 (5):2258-2270. doi:10.1002/hep.30337

12. Finn RS, Qin S, Ikeda M, et al. Atezolizumab plus bevacizumab in unresectable hepatocellular carcinoma. $N$ Engl J Med. 2020;382 (20):1894-1905. doi:10.1056/NEJMoa1915745

13. Finn RS, Qin S, Ikeda M, et al. IMbrave150: updated overall survival (OS) data from a global, randomized, open-label phase III study of atezolizumab (atezo) + bevacizumab (bev) versus sorafenib (sor) in patients (pts) with unresectable hepatocellular carcinoma (HCC). J Clin Oncol. 2021;39(3_suppl):267. doi:10.1200/JCO.2021.39.3_suppl.267

14. Francisco LM, Sage PT, Sharpe AH. The PD-1 pathway in tolerance and autoimmunity. Immunol Rev. 2010;236:219-242.
15. Pardoll DM. The blockade of immune checkpoints in cancer immunotherapy. Nat Rev Cancer. 2012;12(4):252-264. doi:10.1038/ $\operatorname{nrc} 3239$

16. Iñarrairaegui M, Melero I, Sangro B. Immunotherapy of hepatocellular carcinoma: facts and hopes. Clin Cancer Res. 2018;24 (7):1518-1524. doi:10.1158/1078-0432.CCR-17-0289

17. Hanahan D, Weinberg RA. Hallmarks of cancer: the next generation. Cell. 2011;144(5):646-674. doi:10.1016/j.cell.2011.02.013

18. Tripathi A, Debelius J, Brenner DA, et al. The gut-liver axis and the intersection with the microbiome. Nat Rev Gastroenterol Hepatol. 2018;15(7):397-411.

19. Sangro B, Gomez-Martin C, de la Mata M, et al. A clinical trial of CTLA-4 blockade with tremelimumab in patients with hepatocellular carcinoma and chronic hepatitis C. J Hepatol. 2013;59(1):81-88. doi:10.1016/j.jhep.2013.02.022

20. Larkin J, Chiarion-Sileni V, Gonzalez R, et al. Five-year survival with combined nivolumab and ipilimumab in advanced melanoma. $N \mathrm{Engl}$ J Med. 2019;381(16):1535-1546. doi:10.1056/NEJMoa1910836

21. Motzer RJ, Tannir NM, McDermott DF, et al. Nivolumab plus ipilimumab versus sunitinib in advanced renal-cell carcinoma. $N$ Engl J Med. 2018;378(14):1277-1290. doi:10.1056/NEJMoa1712126

22. Waldman AD, Fritz JM, Lenardo MJ. A guide to cancer immunotherapy: from T cell basic science to clinical practice. Nat Rev Immunol. 2020;20(11):651-668.

23. Das R, Verma R, Sznol M, et al. Combination therapy with anti-CTLA4 and anti-PD-1 leads to distinct immunologic changes in vivo. J Immunol. 2015;194(3):950-959. doi:10.4049/jimmunol.1401686

24. Apte RS, Chen DS, Ferrara N. VEGF in signaling and disease: beyond discovery and development. Cell. 2019;176(6):1248-1264. doi:10.1016/j.cell.2019.01.021

25. Faivre S, Rimassa L, Finn RS. Molecular therapies for HCC: looking outside the box. J Hepatol. 2020;72(2):342-352. doi:10.1016/j. jhep.2019.09.010

26. Tian L, Goldstein A, Wang H, et al. Mutual regulation of tumour vessel normalization and immunostimulatory reprogramming. Nature. 2017;544(7649):250-254. doi:10.1038/nature21724

27. Pinter M, Jain RK, Duda DG. The current landscape of immune checkpoint blockade in hepatocellular carcinoma: a review. JAMA Oncol. 2021;7(1):113-123. doi:10.1001/jamaoncol.2020.3381

28. El-Khoueiry AB, Sangro B, Yau T, et al. Nivolumab in patients with advanced hepatocellular carcinoma (CheckMate 040): an open-label, non-comparative, Phase 1/2 dose escalation and expansion trial. Lancet. 2017;389(10088):2492-2502. doi:10.1016/S0140-6736(17)31046-2

29. Kudo M, Matilla A, Santoro A, et al. Checkmate-040: nivolumab (NIVO) in patients (pts) with advanced hepatocellular carcinoma (aHCC) and child-pugh B (CPB) status. J Clin Oncol. 2019;37 (suppl_4):327. doi:10.1200/JCO.2019.37.4_suppl.327

30. Yau T, Park JW, Finn RS, et al. CheckMate 459: a randomized, multi-center phase III study of nivolumab (NIVO) vs sorafenib (SOR) as first-line (1L) treatment in patients (pts) with advanced hepatocellular carcinoma (aHCC). Ann Oncol. 2019;30:v875. doi:10.1093/annonc/mdz394.029

31. Rimassa L, Danesi R, Pressiani T, Merle P. Management of adverse events associated with tyrosine kinase inhibitors: improving outcomes for patients with hepatocellular carcinoma. Cancer Treat Rev. 2019;77:20-28. doi:10.1016/j.ctrv.2019.05.004

32. Edeline J, Yau T, Park J-W, et al. CheckMate 459: health-related quality of life (HRQoL) in a randomized, multicenter phase III study of nivolumab (NIVO) versus sorafenib (SOR) as first-line (1L) treatment in patients (pts) with advanced hepatocellular carcinoma (aHCC). J Clin Oncol. 2020;38(4_suppl):483. doi:10.1200/JCO.2020.38.4_suppl.483

33. Yau T, Zagonel V, Santoro A, et al. Nivolumab (NIVO) + ipilimumab (IPI) + cabozantinib (CABO) combination therapy in patients (pts) with advanced hepatocellular carcinoma (aHCC): results from CheckMate 040. J Clin Oncol. 2020;38(suppl_4):478. doi:10.1200/ JCO.2020.38.4_suppl.478 
34. Zhu AX, Finn RS, Edeline J, et al. Pembrolizumab in patients with advanced hepatocellular carcinoma previously treated with sorafenib (KEYNOTE-224): a non-randomised, open-label Phase 2 trial. Lancet Oncol. 2018;19(7):940-952. doi:10.1016/S1470-2045(18) 30351-6

35. Finn RS, Ryoo BY, Merle P, et al. Pembrolizumab as second-line therapy in patients with advanced hepatocellular carcinoma in KEYNOTE-240: a randomized, double-blind, phase III trial. J Clin Oncol. 2020;38(3):193-202. doi:10.1200/JCO.19.01307

36. Qin S, Finn RS, Kudo M, et al. RATIONALE 301 study: tislelizumab versus sorafenib as first-line treatment for unresectable hepatocellular carcinoma. Future Oncol. 2019;15(16):1811-1822. doi:10.2217/fon2019-0097

37. Desai J, Deva S, Lee JS, et al. Phase IA/IB study of single-agent tislelizumab, an investigational anti-PD-1 antibody, in solid tumors. $J$ Immunother Cancer. 2020;8(1):e000453. doi:10.1136/jitc-2019000453

38. Ren Z, Fan J, Xu J, et al. Sintilimab plus bevacizumab biosimilar vs sorafenib as first-line treatment for advanced hepatocellular carcinoma (ORIENT-32). Ann Oncol. 2020;31:S1287-S1318. doi:10.1016/j.annonc.2020.10.134

39. Finn RS, Ikeda M, Zhu AX, et al. Phase Ib study of lenvatinib plus pembrolizumab in patients with unresectable hepatocellular carcinoma. J Clin Oncol. 2020;38(26):2960-2970. doi:10.1200/ JCO.20.00808

40. Llovet JM, Kudo M, Cheng A-L, et al. Lenvatinib (len) plus pembrolizumab (pembro) for the first-line treatment of patients (pts) with advanced hepatocellular carcinoma (HCC): phase 3 LEAP-002 study. $J$ Clin Oncol. 2019;37(15_suppl):TPS4152. doi:10.1200/ JCO.2019.37.15_suppl.TPS4152

41. Kudo M, Ikeda M, Motomura K, et al. A phase Ib study of lenvatinib (LEN) plus nivolumab (NIV) in patients (pts) with unresectable hepatocellular carcinoma (uHCC): study 117. J Clin Oncol. 2020;38 (suppl_4):513. doi:10.1200/JCO.2020.38.4_suppl.513

42. El-Khoueiry AB, Kim RD, Harris WP, et al. Phase Ib study of regorafenib (REG) plus pembrolizumab (PEMBRO) for first-line treatment of advanced hepatocellular carcinoma (HCC). J Clin Oncol. 2020;38:564. doi:10.1200/JCO.2020.38.4_suppl.564

43. Xu J, Shen J, Gu S, et al. Camrelizumab in combination with apatinib in patients with advanced hepatocellular carcinoma (RESCUE): a nonrandomized, open-label, phase II trial. Clin Cancer Res. 2021;27(4):1003-1011. doi:10.1158/1078-0432.CCR-20-2571

44. Forner A, Da Fonseca LG, Á D-G, Sanduzzi-Zamparelli M, Reig M, Bruix J. Controversies in the management of hepatocellular carcinoma. JHEP Rep. 2019;1(1):17-29. doi:10.1016/j. jhepr.2019.02.003

45. Bruix J, Takayama T, Mazzaferro V, et al. Adjuvant sorafenib for hepatocellular carcinoma after resection or ablation (STORM): a phase 3, randomised, double-blind, placebo-controlled trial. Lancet Oncol. 2015;16(13):1344-1354. doi:10.1016/S14702045(15)00198-9

46. Weber J, Mandala M, Del Vecchio M, et al. Adjuvant nivolumab versus ipilimumab in resected stage III or IV melanoma. $N$ Engl $J$ Med. 2017;377(19):1824-1835. doi:10.1056/NEJMoa1709030

47. Kelly RJ, Ajani JA, Kuzdzal J, et al. Adjuvant nivolumab in resected esophageal or gastroesophageal junction cancer. $N$ Engl $J$ Med. 2021;384(13):1191-1203. doi:10.1056/NEJMoa2032125

48. Kuang M, Peng BG, Lu MD, et al. Phase II randomized trial of autologous formalin-fixed tumor vaccine for postsurgical recurrence of hepatocellular carcinoma. Clin Cancer Res. 2004;10 (5):1574-1579. doi:10.1158/1078-0432.CCR-03-0071

49. Takayama T, Sekine T, Makuuchi M, et al. Adoptive immunotherapy to lower postsurgical recurrence rates of hepatocellular carcinoma: a randomised trial. Lancet. 2000;356(9232):802-807. doi:10.1016/ S0140-6736(00)02654-4
50. Friend BD, Venick RS, McDiarmid SV, et al. Fatal orthotopic liver transplant organ rejection induced by a checkpoint inhibitor in two patients with refractory, metastatic hepatocellular carcinoma. Pediatr Blood Cancer. 2017;64(12):e26682. doi:10.1002/pbc.26682

51. Pinato DJ, Cortellini A, Sukumaran A, et al. PRIME-HCC: phase Ib study of neoadjuvant ipilimumab and nivolumab prior to liver resection for hepatocellular carcinoma. BMC Cancer. 2021;21(1):301. doi:10.1186/s12885-021-08033-x

52. Lee S, Loecher M, Iyer R. Immunomodulation in hepatocellular cancer. J Gastrointest Oncol. 2018;9(1):208-219. doi:10.21037/ jgo.2017.06.08

53. Choi C, Yoo GS, Cho WK, Park HC. Optimizing radiotherapy with immune checkpoint blockade in hepatocellular carcinoma. World $J$ Gastroenterol. 2019;25(20):2416-2429. doi:10.3748/wjg.v25. i2 20.2416

54. Liao J, Xiao J, Zhou Y, Liu Z, Wang C. Effect of transcatheter arterial chemoembolization on cellular immune function and regulatory $\mathrm{T}$ cells in patients with hepatocellular carcinoma. Mol Med Rep. 2015;12(4):6065-6071. doi:10.3892/mmr.2015.4171

55. Singal AG, Hoshida Y, Pinato DJ, et al. International liver cancer association (ILCA) white paper on biomarker development for hepatocellular carcinoma. Gastroenterology. 2021;160:2572-2584. doi:10.1053/j.gastro.2021.01.233

56. Le DT, Uram JN, Wang H, et al. PD-1 blockade in tumors with mismatch-repair deficiency. $N$ Engl $J$ Med. 2015;372 (26):2509-2520. doi:10.1056/NEJMoa1500596

57. Eso Y, Shimizu T, Takeda H, Takai A, Marusawa H. Microsatellite instability and immune checkpoint inhibitors: toward precision medicine against gastrointestinal and hepatobiliary cancers. J Gastroenterol. 2020;55(1):15-26. doi:10.1007/s00535-019-01620-7

58. Ando Y, Yamauchi M, Suehiro Y, et al. Complete response to pembrolizumab in advanced hepatocellular carcinoma with microsatellite instability. Clin J Gastroenterol. 2020;13(5):867-872. doi:10.1007/ s12328-020-01099-3

59. Kawaoka T, Ando Y, Yamauchi M, et al. Incidence of microsatellite instability-high hepatocellular carcinoma among Japanese patients and response to pembrolizumab. Hepatol Res. 2020;50(7):885-888. doi:10.1111/hepr.13496

60. Marabelle A, Fakih M, Lopez J, et al. Association of tumour mutational burden with outcomes in patients with advanced solid tumours treated with pembrolizumab: prospective biomarker analysis of the multicohort, open-label, phase 2 KEYNOTE-158 study. Lancet Oncol. 2020;21 (10):1353-1365. doi:10.1016/S1470-2045(20)30445-9

61. Ang C, Klempner SJ, Ali SM, et al. Prevalence of established and emerging biomarkers of immune checkpoint inhibitor response in advanced hepatocellular carcinoma. Oncotarget. 2019;10(40):4018. doi:10.18632/oncotarget.26998

62. Doroshow DB, Bhalla S, Beasley MB, et al. PD-L1 as a biomarker of response to immune-checkpoint inhibitors. Nat Rev Clin Oncol. 2021;18:345-362. doi:10.1038/s41571-021-00473-5

63. Huang CY, Wang Y, Luo GY, et al. Relationship between PD-L1 expression and CD8+ T-cell immune responses in hepatocellular carcinoma. $J$ Immunother. 2017;40(9):323-333. doi:10.1097/ CJI.0000000000000187

64. Sia D, Jiao Y, Martinez-Quetglas I, et al. Identification of an immune-specific class of hepatocellular carcinoma, based on molecular features. Gastroenterology. 2017;153(3):812-826. doi:10.1053/ j.gastro.2017.06.007

65. Sangro B, Melero I, Wadhawan S, et al. Association of inflammatory biomarkers with clinical outcomes in nivolumab-treated patients with advanced hepatocellular carcinoma. $J$ Hepatol. 2020;73 (6):1460-1469. doi:10.1016/j.jhep.2020.07.026

66. Pfister D, Nunez NG, Pinyol R, et al. NASH limits anti-tumour surveillance in immunotherapy-treated HCC. Nature. 2021;592:450-456. doi:10.1038/s41586-021-03362-0 
67. Kelley RK, Greten TF, Phimister EG. Hepatocellular carcinoma origins and outcomes. $N$ Engl $J$ Med. 2021;385:280-282. doi:10.1056/NEJMcibr2106594

68. Hills RK. Non-inferiority trials: No better? No worse? No change? No pain? Br J Haematol. 2017;176(6):883-887. doi:10.1111/bjh.14504

69. Fessas P, Kaseb A, Wang Y, et al. Post-registration experience of nivolumab in advanced hepatocellular carcinoma: an international study. J Immunother Cancer. 2020;8(2):e01033. doi:10.1136/jitc2020-001033

70. Beaver JA, Pazdur R. "Dangling" accelerated approvals in oncology. N Engl J Med. 2021;384(18):e68. doi:10.1056/NEJMp2104846

71. Ruiz de Galarreta M, Bresnahan E, Molina-Sánchez $P$, et al. $\beta$-catenin activation promotes immune escape and resistance to anti-PD-1 therapy in hepatocellular carcinoma. Cancer Discov. 2019;9 (8):1124-1141. doi:10.1158/2159-8290.CD-19-0074
72. Pinato DJ, Fessas P, Sapisochin G, Marron TU. Perspectives on the neoadjuvant use of immunotherapy in hepatocellular carcinoma. Hepatology. 2020;74(1);483-490. doi:10.1002/hep.31697

73. Brown ZJ, Greten TF, Heinrich B. Adjuvant treatment of hepatocellular carcinoma: prospect of immunotherapy. Hepatology. 2019;70 (4):1437-1442. doi:10.1002/hep.30633

74. Giaj Levra M, Cotté FE, Corre R, et al. Immunotherapy rechallenge after nivolumab treatment in advanced non-small cell lung cancer in the real-world setting: a national data base analysis. Lung Cancer. 2020;140:99-106. doi:10.1016/j.lungcan.2019.12.017

75. Gobbini E, Charles J, Toffart AC, Leccia MT, Moro-Sibilot D, Giaj Levra M. Current opinions in immune checkpoint inhibitors rechallenge in solid cancers. Crit Rev Oncol Hematol. 2019;144:102816. doi:10.1016/j.critrevonc.2019.102816

\section{Publish your work in this journal}

The Journal of Hepatocellular Carcinoma is an international, peerreviewed, open access journal that offers a platform for the dissemination and study of clinical, translational and basic research findings in this rapidly developing field. Development in areas including, but not limited to, epidemiology, vaccination, hepatitis therapy, pathology and molecular tumor classification and prognostication are all considered for publication. The manuscript management system is completely online and includes a very quick and fair peer-review system, which is all easy to use. Visit http://www.dovepress.com/ testimonials.php to read real quotes from published authors. 